\title{
Molecular response of gastrointestinal stromal tumour after treatment with tyrosine kinase inhibitor imatinib mesylate
}

\author{
P Reichardt, U Schneider, C Stroszczynski, D Pink, P Hohenberger
}

J Clin Pathol 2004;57:215-217. doi: 10.1136/¡cp.2004.11379

\begin{abstract}
Bleeding from the tumour site is not uncommon during the treatment of gastrointestinal stromal tumours with imatinib mesylate. It might represent an early reaction of highly vascularised tumour tissue to receptor blockade. Although often requiring emergency surgery, this is not necessarily a deleterious sign. Slow tumour regression and cystic tissue alteration may follow. Using immunohistochemistry and consecutive resection specimens, it was shown that the number of mitoses decreased significantly and MIB-1 as a marker of cell proliferation could no longer be detected. In the few tumour cells still present, the magnitude of expression of the pathognomonic marker CD117 remained unchanged. Decreases in the size of tumours responding to imatinib mesylate cannot be expected to meet the World Health Organisation or RECIST (response evaluation criteria in solid tumours) criteria. This underlines the necessity of functional imaging by positron emission tomography, contrast enhanced magnetic resonance imaging, or magnetic resonance spectroscopy to assess the response to treatment.
\end{abstract}

l matinib mesylate (STI571), a small molecule tyrosine kinase inhibitor, is a highly effective new treatment strategy for gastrointestinal stromal tumours (GISTs), which acts through the inhibition of c-kit, a cellular tyrosine kinase receptor uniformly expressed by GISTs. ${ }^{12}$ Because of its unique mode of action and in contrast to conventional chemotherapy, treatment with imatinib does not result in rapid tumour regression. Few data exist on the histological changes during patient treatment with imatinib mesylate. ${ }^{2}$ We obtained representative serial tissue specimens from a patient requiring three consecutive emergency laparotomies for intraabdominal bleeding episodes during imatinib treatment for a GIST, and evaluated the resection specimens conventionally and by immunohistochemistry (IHC).

\begin{abstract}
"Because of its unique mode of action and in contrast to conventional chemotherapy, treatment with imatinib does not result in rapid tumour regression"
\end{abstract}

\section{CASE-REPORT}

A 44 year old man was first diagnosed as suffering from a malignant GIST of the small bowel in December 1999. The tumour was resected and the patient treated with four cycles of adjuvant chemotherapy, including doxorubicin and ifosfamide. Liver metastases were diagnosed five months later and laser induced thermotherapy was performed, followed by atypical liver resection. Another six months later, peritoneal and recurrent liver metastases were diagnosed and the patient received carboplatinum and dacarbacine chemotherapy, resulting in stable disease for three months. Thereafter, magnetic resonance imaging (MRI) showed tumour progression, with multiple $(>100)$ peritoneal seedings (up to $13 \mathrm{~cm}$ in diameter) and liver metastases (up to $11 \mathrm{~cm}$; fig $1 \mathrm{~A}$ ), and the patient was referred for further treatment in April 2001. As a result of severe abdominal pain, weight loss of $10 \mathrm{~kg}$ within the past two months, anaemia (haemoglobin $(\mathrm{Hb}), 85 \mathrm{~g} / \mathrm{litre}$ ), and fatigue his Karnofsky index was 60 . Treatment with imatinib mesylate was started at a dose of $800 \mathrm{mg}$ daily.

After two weeks of treatment his general condition improved greatly. The main side effects of treatment were nausea and emesis, in addition to loss of appetite, and he repeatedly required blood transfusions. MRI documented a partial remission of indicator lesions after two months of treatment. Former solid metastases had regressed to cystic structures with a small parenchymic wall (fig 1B). Two weeks later, the patient had to be hospitalised acutely because of massive emesis, dizziness, and severe abdominal pain. His haemoglobin value had decreased from $110 \mathrm{~g} /$ litre to $67 \mathrm{~g} /$ litre within 24 hours, although his platelet count was normal. Abdominal ultrasound demonstrated the presence of 4-5 litres of ascites, shown to be haemorrhagic by paracentesis (Hb, 52 g/litre). Upon emergency laparotomy, we found numerous peritoneal tumour nodules, which were dark blue in colour, hypervascularised, and full of blood. More than 2 litres of haemorrhagic ascites was removed, which was thought to derive from the rupture of tumour nodules. Several ruptured nodules were resected. Postoperatively, the patient stabilised rapidly and imatinib treatment was restarted at a dose of $600 \mathrm{mg} /$ day. Eight weeks later, a second emergency laparotomy had to be performed because of recurrent abdominal bleeding under similar circumstances. Again, haemorrhagic ascites was removed and several ruptured tumour nodules were resected; the patient recovered quickly. At this time, no further regression of tumour size was seen in MRI. Four weeks later, a third laparotomy became necessary for abdominal bleeding. This time, only a few interenteric nodules could be detected, most of which were pale, slack cysts with some gelatinous contents. The abdominal cavity, except for the liver, was cleared of more than $95 \%$ of the tumour remnants. The patient continues on imatinib treatment and his $\mathrm{Hb}$ is stable at $110 \mathrm{~g} /$ litre and he has a Karnofsky index of 90. The most recent follow up showed residual cystic liver lesions and single peritoneal deposits.

Tumour tissue specimens obtained before the start of treatment and during the laparotomies were stained by haematoxylin and eosin and by IHC for CD117 and CD34. Proliferative activity was assessed by counting mitoses in 50

Abbreviations: GIST, gastrointestinal stromal tumour; $\mathrm{Hb}$, haemoglobin; IHC, immunohistochemistry; MRI, magnetic resonance imaging 


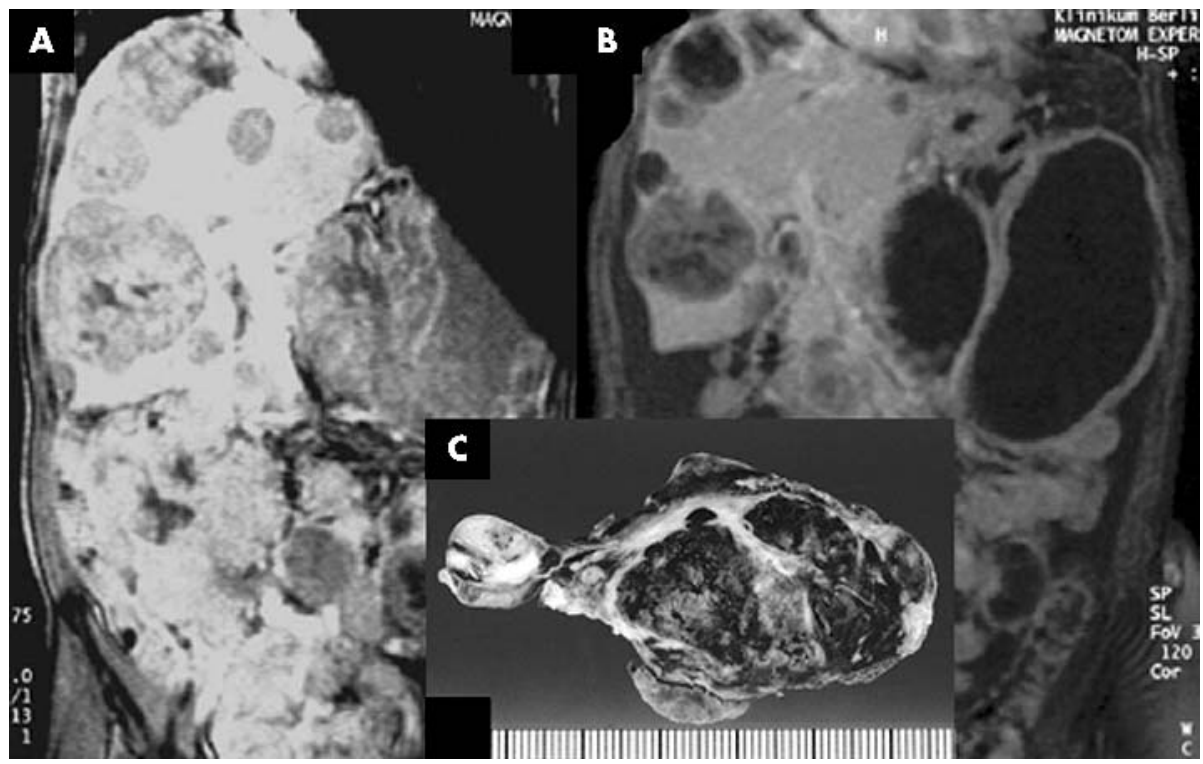

Figure 1 Changes of liver and peritoneal metastases as assessed by magnetic resonance tomography during treatment with imatinib. Solid metastases change from $(A)$ totally solid lesions to (B) a cystic appearance with thin, vascularised tumour boundaries and (C) central haemorrhagia.

high power fields. Before treatment with imatinib, histology showed a typical predominantly spindle cell GIST with heterogeneous nuclear atypia and a mitotic activity averaging 18 mitoses/50 high power fields (fig $2 \mathrm{~A}$ ). At the time of the first surgical intervention, there was some decrease of cellular cohesion, with shrinkage of the tumour tissue and widespread haemorrhagia, but no necrosis (figs 1C, 2B). No mitoses were seen and IHC revealed a proliferative fraction of almost zero, as determined by staining for MIB-1. From the second to the third laparotomy, the now paucicellular tumour tissue became increasingly gelatinous. In some areas viable, cellular, and once again non-proliferating tumour tissue remained (fig 2C, D). In contrast, the intensity of staining for CD117 and CD34 showed no change throughout the course of disease.

\section{DISCUSSION}

Imatinib mesylate has changed the treatment of metastatic GISTs completely. Recent data report that $54 \%$ of patients develop a partial remission and another $28 \%$ gain stable disease under treatment. ${ }^{3}$ The toxicity profile of the drug mainly shows nausea, diarrhoea, oedema, or dermatitis. Despite the general impression of imatinib being a relatively safe and well tolerated drug, lethal complications can still occur. In particular, those patients with very large tumours are at risk for major bleeding, and bleeding can occur repeatedly at any time of treatment. Bleeding episodes were reported at an incidence of $5 \%,{ }^{3}$ but do not preclude a successful course of disease. The question of whether haemorrhage with normal platelet counts is dose dependent cannot be answered yet.

Bleeding can occur outside of the abdominal cavity, such as in liver metastases, resulting in a hepatic haematoma. In this context, our observation of repetitive bleeding is of interest to separate treatment related side effects from symptoms related to treatment efficacy. Bleeding during treatment with imatinib may cause an increase in the size of measurable lesions, which might be misinterpreted as a progression, even though these changes might indicate a response to treatment. Thus, standard response evaluation criteria, such as those

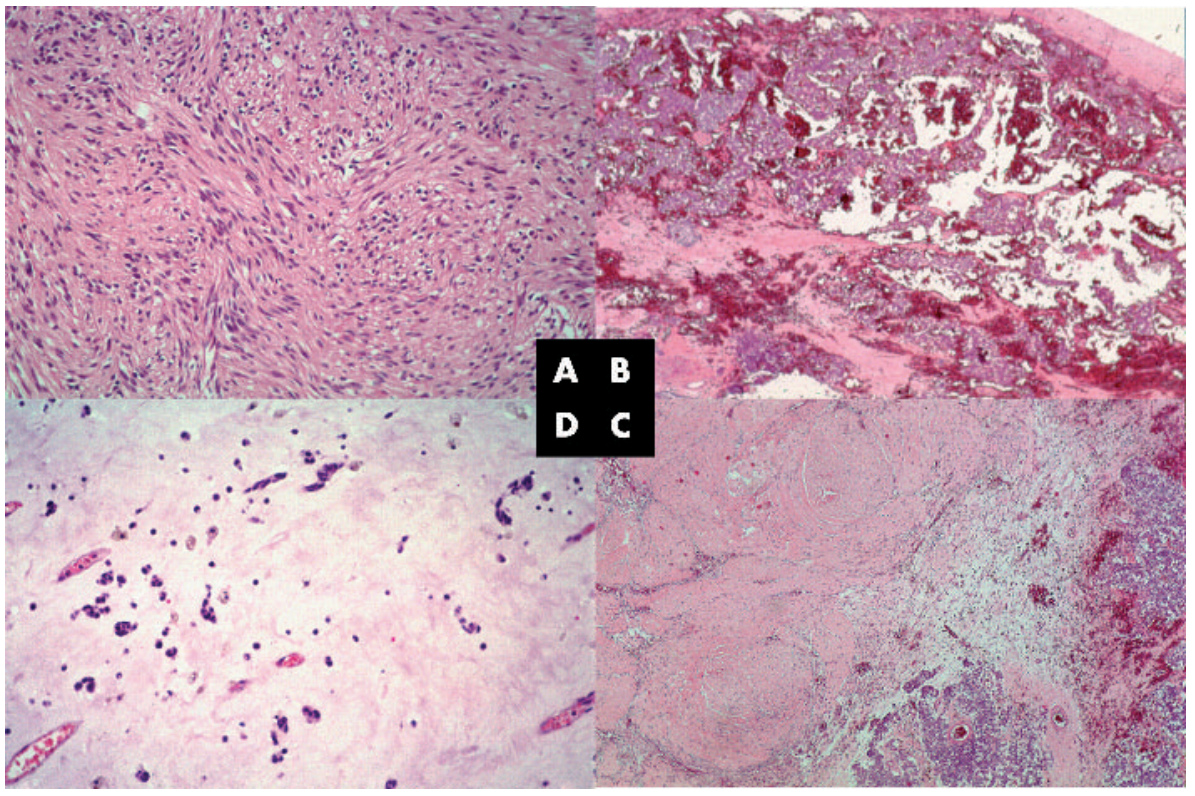

Figure 2 (A) Typical predominantly spindle cell gastrointestinal stromal tumour with heterogeneous nuclear atypicality and a mitotic activity averaging 18 mitoses/50 high power fields (original magnification, $\times 200$ ). (B) Intratumorous haemorrhagia but no necrosis at the time of the first surgical intervention (original magnification, $\times 40$ ), with (C) a decrease in cellular cohesion, shrinkage of the tumour tissue, and widespread haemorrhagia (original magnification, $\times 40$ ). No mitoses were seen, and immunohistochemistry revealed a proliferative fraction of almost zero, as determined by staining for MIB-1. (D) The gelatinous changes to the paucicellular tumour tissue remained, and tumour tissue was still nonproliferating (original magnification, $\times 200$ ). All slides were stained with haematoxylin and eosin. 


\section{Take home messages}

- We report a patient with a gastrointestinal stromal tumour who was treated with imatinib mesylate and who suffered from repeated episodes of bleeding from the tumour site

- Immunohistochemistry on consecutive resection specimens showed that the number of mitoses decreased significantly and the MIB-1 marker of cell proliferation was no longer detected

- Thus, these bleeding episodes were interpreted as a response to treatment rather than a side effect of treatment

- Decreases in the size of tumours responding to imatinib mesylate cannot be expected to meet the World Health Organisation or RECIST (response evaluation criteria in solid tumours) criteria

- Functional imaging by positron emission tomography, contrast enhanced magnetic resonance imaging, or magnetic resonance spectroscopy should be used to assess the treatment response

- Treatment with imatinib should be prolonged

recommended by the World Health Organisation or RECIST (response evaluation criteria in solid tumours), may be inappropriate to evaluate early treatment effects. Functional 18-FDG positron emission tomography has been used successfully to demonstrate response to treatment as early as 24 to 48 hours after the start of treatment. ${ }^{35}$ Contrast enhanced MRI was used to assess the extent of tumour necrosis in the pre-imatinib era, ${ }^{6}$ and to characterise tumours before starting treatment with imatinib. ${ }^{7}$ Our patient and a previous report demonstrate cystic changes in hepatic metastases and effectively mirror response to treatment. ${ }^{8}$ Even the analysis of Houndsfield units might be of distinct value provided contrast media are given. Magnetic resonance spectroscopy might be an additional tool to evaluate the transformation of tumour nodules into cystic, non-proliferative remnants. ${ }^{9}$

"Our observation of repetitive bleeding is of interest to separate treatment related side effects from symptoms related to treatment efficacy"

Histologically, ongoing loss of mitotic activity was seen in the resection specimens that were consecutively removed during the first six months of treatment. The tumour underwent pronounced regression and then remained stable in size in radiological aspects. However, despite the macroscopic cyst-like appearance, CD117 and CD34 expression was found at all times in the examined specimens. In experiments using dermatofibrosarcoma protuberans cell lines, imatinib showed growth inhibitory effects predominantly through the induction of tumour cell apoptosis. ${ }^{10}$ Thus, "dormant" GIST cells might persist, which harbour the potential for disease relapse. These findings underline the necessity of prolonged treatment with imatinib, as does the observation of late recurrences, sometimes after more than two years of successful tumour control.

\section{Authors' affiliations}

P Reichardt, D Pink, Division of Medical Oncology, Hematology, and Tumour Immunology, Robert Roessle Hospital and Tumour Institute,

Charité University Hospital, The Humboldt University at Berlin, D-13122

Germany

U Schneider, Pathology Laboratory, Robert Roessle Hospital and Tumour Institute

C Stroszczynski, Department of Radiodiagnostics, Robert Roessle Hospital and Tumour Institute

P Hohenberger, Division of Surgery and Surgical Oncology, Robert

Roessle Hospital and Tumour Institute

Correspondence to: Professor P Hohenberger, Division of Surgery and Surgical Oncology, Charité, University Medicine Berlin, Campus BerlinBuch, Lindenberger Weg 80, D-13122 Berlin, Germany;

hohenberger@rrk-berlin.de

Accepted for publication 13 August 2003

\section{REFERENCES}

1 Miettinen M, Sarloma-Rikala M, Lasota J. Gastrointestinal stromal tumors: recent advances in understanding of their biology. Hum Pathol 1999;30:1213-20.

2 Joensuu H, Roberts PJ, Sarlomo-Rikala M, et al. Effect of the tyrosine kinase inhibitor STI571 in a patient with a metastatic gastrointestinal stromal tumor. N Engl J Med 2001;344:1052-6.

3 van Oosterom AT, Judson I, Verweij J, et al. Safety and efficacy of imatinib (STI571) in metastatic gastrointestinal stromal tumours: a phase I study. Lancet 2001;358:1421-3.

4 Demetri GD, von Mehren M, Blanke CD, et al. Efficacy and safety of imatinib mesylate in advanced gastrointestinal stromal tumors. N Engl J Med 2002;347:472-80.

5 Joensuu H, Fletcher $C$, Dimitrijevic S, et al. Management of malignant gastrointestinal stromal tumours. Lancet Oncol 2002;3:655-64.

6 Hasegawa S, Semelka RC, Noone TC, et al. Gastric stromal sarcomas: correlation of MR imaging and histopathologic findings in nine patients. Radiology 1998;208:591-5.

7 Jost D, Stroszczynski C, Chmelik P, et al. Morphology of gastrointestinal stromal tumors in advanced stages of the disease: baseline findings before chemotherapy with imatinib. Rofo Fortschr Geb Rontgenstr Neven Bildgeb Verfahr 2003;175:791-8.

8 Chen MYM, Bechtold RE, Savage PD. Cystic changes in hepatic metastases from gastrointestinal stromal tumors (GISTs) treated with gleevec (imatinib mesylate). AJR Am J Roentgenol 2002;179:1059-62.

9 Kettelhack C, von Wickede M, Vogl TJ, et al. Histological tumor response predicted by 31P-magnetic resonance spectroscopy (31P-MRS) following isolated limb perfusion for soft tissue sarcoma. Cancer 2002;94:1557-64.

10 Sjoblom T, Shimizu A, O'Brien KP, et al. Growth inhibition of dermatofibrosarcoma protuberans tumors by the platelet-derived growth factor receptor antagonist STI571 through induction of apoptosis. Cancer Res $2001 ; 61: 5778-83$. 